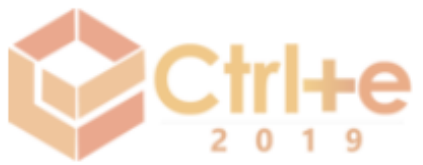

\title{
Divertix: um Recurso Educacional Digital para a Aprendizagem da Estatística nos Anos Iniciais do Ensino Fundamental
}

\author{
Joyce da Silva Sousa 1, Lídya de Lima Monteiro 1, Antonio Nathan Serra Souza 1, \\ Danilo do Carmo de Souza 1, Juscileide Braga de Castro1, Márcia Duarte Medeiros1, \\ Maria de Fátima Costa de Souza1, José Aires de Castro Filho1
}

1Instituto UFC Virtual - Grupo de Pesquisa e Produção de Ambientes Interativos e Objetos de Aprendizagem (Proativa) - Universidade Federal do Ceará (UFC) - Cep: 60440-554 Fortaleza - CE - Brasil

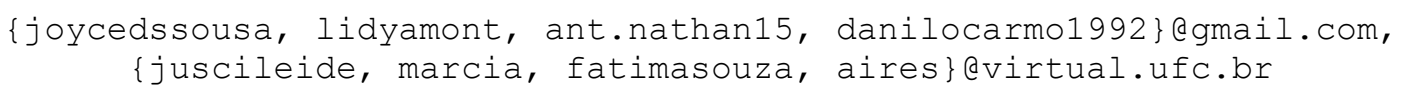

\begin{abstract}
Although Statistics is part of school curriculum since early years of elementary school, most students present difficulties concerning the understanding of numerical or textual data and information expressed in graphs or tables. Within this, the objective of this work is to present the development of a Digital Educational Resource (RED) and its contributions to teaching of statistical concepts. The RED has a narrative that develops in a magazine scene (Divertix) where, after an incident, the student is invited to use statistical and informational skills to restore the journal's contents and discover clues that reveal the identity of the invader.
\end{abstract}

Resumo. Ainda que a Estatística faça parte do currículo escolar desde os anos iniciais do Ensino Fundamental, a grande parte dos estudantes apresentam dificuldades concernentes à compreensão de dados e informações numéricas ou textuais expressas em gráficos ou tabelas. Dentro desta perspectiva o objetivo deste trabalho é apresentar o desenvolvimento de um Recurso Educacional Digital (RED) e suas contribuições para o ensino de conceitos estatísticos. O RED possui narrativa que se desenvolve no cenário de uma revista (Divertix) em que o usuário é convidado a utilizar habilidades referentes à Estatística, como ler e interpretar gráficos e tabela, relacionar texto e gráfico e construir gráficos; para finalizar as matérias da revista.

\section{Introdução}

Vivemos em um tempo marcado por um fluxo contínuo de informações. Cotidianamente, as crianças têm contato com dados estatísticos por meio de jornais, revistas, sites, blogs, entre outros. Grandes quantidades de dados estão dispostos e são apontados em tabelas e gráficos difundidos pelos diversos meios de comunicação. Nesse sentido, é imprescindível que os estudantes aprendam em sala de aula a compreender e a interpretar os conceitos estatísticos, mobilizando suas diferentes representações. Assim, viver em momentos atuais requer a percepção de um cidadão crítico e que consiga avaliar, opinar e argumentar estatisticamente relacionando dados e contextos [Gal 2002].

No cenário educacional brasileiro, a Estatística ganha força a partir da implantação dos Parâmetros Curriculares Nacionais (PCN) de Matemática que a ressalta em um dos 
cinco blocos de conteúdos, a saber: Tratamento da Informação [Brasil 1997]. Atualmente, com a Base Nacional Comum Curricular (BNCC), a Estatística recebe mudanças significativas. A BNCC estabelece como essencial o desenvolvimento de habilidades relacionadas à coleta, à organização, à representação, à interpretação e análise de dados em variados contextos, atrelando a estas a capacidade do aluno de fazer julgamentos fundamentados e tomar decisões adequadas [Brasil 2017a].

Apesar da Estatística ser indicada a fazer parte do currículo escolar, desde os anos iniciais do Ensino Fundamental, a população brasileira, de uma maneira geral, apresenta dificuldades relacionadas com a compreensão de dados. Sobre isso, o Indicador Nacional de Alfabetização Funcional (INAF), em pesquisa mais recente realizada com a população adulta do Brasil com idade de 15 a 64 anos, revelou que cerca de $30 \%$ dos investigados estão abaixo da proficiência elementar, o que significa que não são capazes de comparar ou relacionar "informações numéricas ou textuais expressas em gráficos ou tabelas simples, envolvendo situações de contexto cotidiano doméstico ou social; sendo considerados, segundo a classificação da pesquisa, como analfabetos funcionais [Inaf 2018, p. 21]. A pesquisa do Inaf $(2018$, p. 21$)$ também constata que apenas $12 \%$ são proficientes, sendo portanto capazes de interpretar "tabelas e gráficos envolvendo mais de duas variáveis, compreendendo elementos que caracterizam certos modos de representação de informação quantitativa (escolha do intervalo, escala, sistema de medidas ou padrões de comparação)".

Por outro lado, os resultados de avaliações externas como do Sistema de Avaliação da Educação Básica (SAEB) apontam que estudantes da Educação Básica possuem dificuldades em conceitos elementares. Considerando os estudantes do $5^{\circ}$ ano do Ensino Fundamental, na escala de proficiência, encontram-se no nível 4 de um total de 10, ou seja, possuem aprendizagem em conteúdos básicos [Brasil 2017b]. No tocante aos conteúdos relacionados à Estatística, isso se reflete nas capacidades de: reconhecer o maior valor em uma tabela cujos dados possuem até oito ordens; localizar um dado em tabelas de dupla entrada. Ademais, somente $15,52 \%$ dos estudantes do $5^{\circ}$ ano estão no nível apontado como adequado. As dificuldades dos alunos e da população adulta em assimilar os conceitos estatísticos e sua aplicabilidade em situações corriqueiras têm levado os pesquisadores em Educação Matemática a desenvolver metodologias e ferramentas que possibilitem o desenvolvimento e aprimoramento dos conceitos dessa área do conhecimento. Uma das possibilidades é a utilização das Tecnologias Digitais da Informação e Comunicação (TDIC). Tendo em vista a crescente expansão das TDIC, evidencia-se a aplicação desses artefatos em sala de aula como potencial para a construção de conteúdos curriculares, em especial os conceitos estatísticos [Castro 2012; Castro e Castro-Filho 2015].

Em pesquisa realizada por Castro (2012) foram planejadas um conjunto de atividades baseadas no uso de RED e de um $b \log _{1}$ a fim de verificar como o uso das TDIC pode contribuir com a aprendizagem de conceitos relacionados ao Tratamento da Informação, como a construção e a interpretação de gráficos de barras e de setores. Dentre os resultados, a pesquisadora apresenta evidências das contribuições das tecnologias, como: a) Representar as informações de situações-problema e situações reais em gráficos construídos com rapidez e facilidade; b) Visualizar e simular diferentes situações com rapidez, permitindo ao aluno compreender as relações existentes entre a frequência, a categoria e o todo, por exemplo; c) Tratar a informação, desde a coleta de dados à comunicação dos resultados; d) Trabalhar com situações reais e com contextos investigativos em atividades colaborativas, envolvendo a produção de dados e informações; e) Explorar os dados produzidos em contextos investigativos, verificando os dados a partir 
de diversas representações, permitindo que os estudantes focassem a interpretação e os conceitos. [Castro 2012, p.154].

O potencial das TDIC também foi relatado no trabalho de Castro e Castro-Filho (2015) que observou o desenvolvimento do pensamento estatístico2, a partir de um processo investigativo realizado em quatro etapas: planejamento, coleta de dados, organização de dados e publicação da notícia. As TDIC foram utilizadas em todas as etapas e possibilitaram a compreensão do processo de coleta de dados, de classificação, de escolha da amostra, do cruzamento de variáveis e da indicação do tipo de gráfico.

Considerando o potencial das TDIC para o trabalho de Estatística e a pouca disponibilização de RED que abordem as habilidades previstas pela BNCC para esta unidade temática, o objetivo deste trabalho é apresentar o desenvolvimento de um Recurso Educacional Digital (RED) intitulado Divertix e suas contribuições para o ensino de conceitos estatísticos. Esse artigo está dividido em quatro seções: as reflexões introdutórias, o processo de desenvolvimento, a apresentação e análise do RED, seguidas das considerações finais.

\section{Processo de Desenvolvimento}

O RED Divertix foi desenvolvido por uma equipe multidisciplinar formado por graduandos de Matemática e de Sistemas e Mídias Digitais, mestrandos em Educação, professores da graduação e pós-graduação, bem como profissionais das áreas de design e de programação, todos vinculados ao Grupo Mídias Digitais na Educação (Mide). Os integrantes constituíram duas equipes, as quais trabalhavam simultaneamente: A primeira equipe, denominada equipe pedagógica, ficou responsável pela criação dos contextos, atividades, textos, narrativas e incorporação dos elementos de gamificação além dos conceitos matemáticos e dos feedbacks de interação com o usuário. Já a segunda equipe, denominada equipe técnica, ficou encarregada das proposições de elementos gráficos, sonoros, fluidez das interações e ferramentas de programação.

Para uma melhor organização, os trabalhos desenvolvidos pelas equipes pedagógica e técnica, foram organizados em um processo de desenvolvimento, composto por 6 etapas, que utilizou como base a perspectiva do modelo iterativo e incremental [Sommerville 2011], conforme Figura 1.

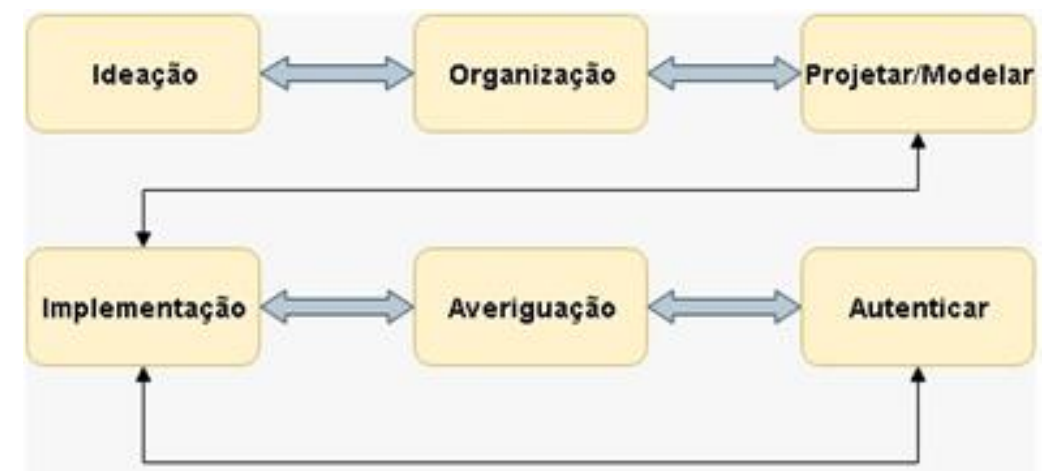

Figura 01. Processo de Desenvolvimento Divertix (Fonte: autores)

A primeira etapa consistiu da Ideação, onde se fez uso da criatividade para geração de ideias, bem como a definição de aspectos relacionados às habilidades e competências de

2 Pensamento estatístico é o "ato de pensar e fazer predições sobre o processo investigativo, inferindo os resultados a partir das mudanças no processo, desde a definição da amostra até a representação dos resultados" [Castro; Castro-Filho 2015. p. 873] 
acordo com as diretrizes da BNCC, o conteúdo a ser trabalhado e os possíveis elementos de gamificação a serem inseridos. Durante essa etapa foram definidos os aspectos centrais do RED, tais como seus objetivos pedagógicos, conteúdos e habilidades a serem contemplados, breve descrição do recurso e público-alvo que deveria atingir. No que tange aos aspectos da gamificação, o foco consistiu em analisar quais elementos do jogo poderiam potencializar o interesse do usuário pela temática abordada, como por exemplo a narrativa, os desafios propostos, a temporização como forma de injetar dinamismo ao recurso, bonificações e os feedbacks instantâneos.

A segunda etapa consistiu da estruturação do RED a ser desenvolvido no intuito de definir aspectos relacionados à representatividade, contextualização e selecionar os elementos de gamificação a serem incorporados no projeto de desenvolvimento para se atingir objetivos específicos.

$\mathrm{Na}$ terceira etapa buscou-se projetar/modelar sequências de navegabilidade, elementos de interação e gamificação. Finalizada a terceira etapa, iniciamos a etapa de implementação com o intuito de produzir um executável. Na etapa de implementação, três linguagens principais foram utilizadas, sendo estas: HTML5 (marcação), JavaScript (programação) e CSS (estilo). Foi utilizado como framework para desenvolvimento o AngularJS, mais especificamente o modelo angular-webpack-config, que facilitou a programação ao dividi-la em estados bem definidos. Para desenvolvimento foi utilizado também o $S A S S$, uma linguagem que pré-processa o $C S S$, ajudando na organização do estilo e diminuindo o tamanho dos arquivos que eram modificados durante o desenvolvimento, ao passo que os reunia quando partiam para a produção. Feito isso, a próxima etapa é de averiguação. Essa etapa, pode ser chamada também de verificação, pois nela se verifica a sequência de navegabilidade, os elementos de interação e se os objetivos definidos no início desse processo são atendidos. Por fim, a etapa de autenticação que corresponde a etapa de validação, que consiste em avaliar se os objetivos pedagógicos foram atingidos da forma como foi projetado. Para dar uma visão mais clara sobre o resultado final. Na seção seguinte será apresentado o RED Divertix.

\section{O RED Divertix}

O Divertix 3 é um RED interdisciplinar destinado para estudantes do $5^{\circ}$ ano do Ensino Fundamental. Ele foi desenvolvido para explorar, preferencialmente, à aprendizagem de habilidades da área de Matemática, pertencentes às unidades temáticas de Probabilidade e Estatística e Números [Brasil 2017a]. Além disso, também contempla habilidades da área de Língua Portuguesa, conforme pode-se observar no Quadro 1.

Quadro 1. Habilidades de Matemática e Língua Portuguesa de acordo com a BNCC

\begin{tabular}{|l|l|}
\hline Habilidades & Descrição das habilidades de Matemática \\
\hline EF05MA07 & $\begin{array}{l}\text { Resolver e elaborar problemas de adição e subtração com números naturais e com } \\
\text { números racionais, cuja representação decimal seja finita, utilizando estratégias } \\
\text { diversas, como cálculo por estimativa, cálculo mental e algoritmos. }\end{array}$ \\
\hline EF05MA08 & $\begin{array}{l}\text { Resolver e elaborar problemas de multiplicação e divisão com números naturais e } \\
\text { com números racionais cuja representação decimal é finita (com multiplicador } \\
\text { natural e divisor natural e diferente de zero), utilizando estratégias diversas, como } \\
\text { cálculo por estimativa, cálculo mental e algoritmos. }\end{array}$ \\
\hline EF05MA24 & Interpretar dados estatísticos apresentados em textos, tabelas e gráficos (colunas ou \\
\hline
\end{tabular}

3 Vídeo de demonstração do recurso disponível em: https://inyurl.com/divertix2019ctrle 


\begin{tabular}{|l|l|}
\hline & $\begin{array}{l}\text { linhas), referentes a outras áreas do conhecimento ou a outros contextos, como saúde } \\
\text { e trânsito, e produzir textos com o objetivo de sintetizar conclusões. }\end{array}$ \\
\hline Habilidades & Descrição das habilidades de Língua Portuguesa \\
\hline EF15LP18 & Relacionar texto com ilustrações e outros recursos gráficos \\
\hline EF05LP23 & Comparar informações apresentadas em gráficos ou tabelas. \\
\hline
\end{tabular}

O RED pode ser classificado como interdisciplinar em relação à área de linguagens, pois aborda em suas atividades, situações nas quais oportuniza-se o desenvolvimento de habilidades na leitura de diversas representações semióticas como textos, imagens e gráficos. Como forma de explorar as habilidades propostas e o engajamento do usuário, o RED possui narrativa que se desenvolve no prédio de uma revista chamada Divertix. Na véspera da publicação de mais uma edição da revista, o prédio é invadido durante a madrugada por um vilão misterioso. No dia seguinte, descobre-se que algumas matérias que estariam nessa edição foram prejudicadas. Nesse contexto, o jogador é convidado a restaurar as matérias da revista, de modo que a edição seja publicada a tempo, além de descobrir pistas que revelem a identidade do invasor. Para resolver esse desafio o jogador deverá cumprir três atividades. Cada uma delas corresponde, respectivamente, às funções de revisor, redator e designer, funções normalmente executadas em uma revista. Cada atividade tem um personagem-guia distinto que desempenha, respectivamente, alguma das funções citadas. As atividades são realizadas por andar-setor do prédio. A primeira atividade possui como cenário o setor de revisão (Figura 02).

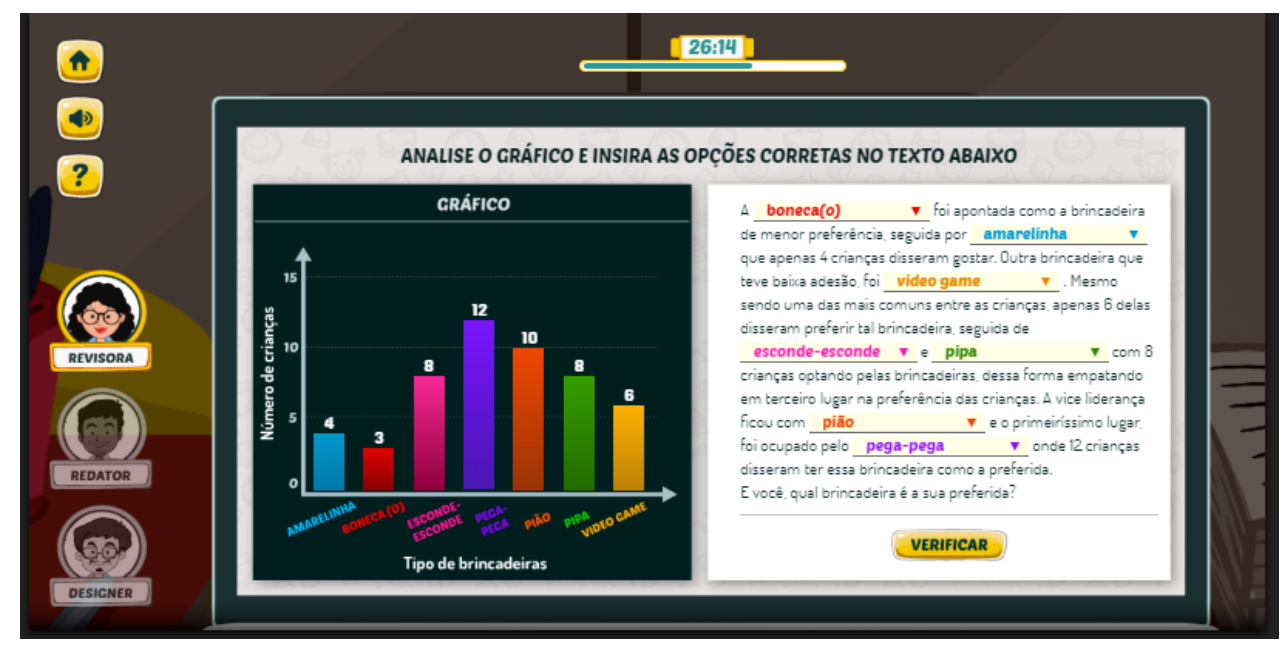

Figura 02. Atividade da Revisora

Após uma breve explicação da função do revisor dada pela personagem, ela contextualiza que uma das matérias precisa da habilidade do revisor para ser recuperada. Então, o texto de uma das matérias será exibido para o jogador. A matéria trata-se de uma pesquisa sobre as brincadeiras preferidas de crianças com idade semelhante às do públicoalvo do RED, entre 9 e 10 anos, com o objetivo de proporcionar a aproximação do jogador com o conteúdo do texto. Após a leitura o jogador é notificado que possui quarenta minutos para terminar todas as atividades e publicar a revista a tempo. O tempo limite foi inserido como estratégia de imersão para que os jogadores compreendam a urgência de entregar a revista no prazo. Nessa primeira atividade, o jogador deverá analisar os dados da pesquisa, que são os votos das crianças em cada brincadeira, apresentados em um gráfico de colunas e 
comparar com o texto que explica os valores que cada coluna representa. Analisando o gráfico, o jogador deverá indicar no texto a brincadeira a qual o dado numérico se refere, pois, eventualmente, algumas brincadeiras estarão indicadas no local incorreto do texto, resultado do trabalho do vilão. A quantidade de votos relacionada a cada coluna é inserida de maneira randômica. Assim, quando o usuário jogar novamente terá que indicar novas respostas para cada campo a ser preenchido. Após a finalização da atividade do Revisor, o jogador recupera uma parte da matéria e encontra uma pista da identidade do vilão. $\mathrm{O}$ próximo passo é subir para o andar acima e realizar a atividade do Redator, em que é apresentada uma matéria sobre as frutas preferidas pelas crianças (Figura 03).

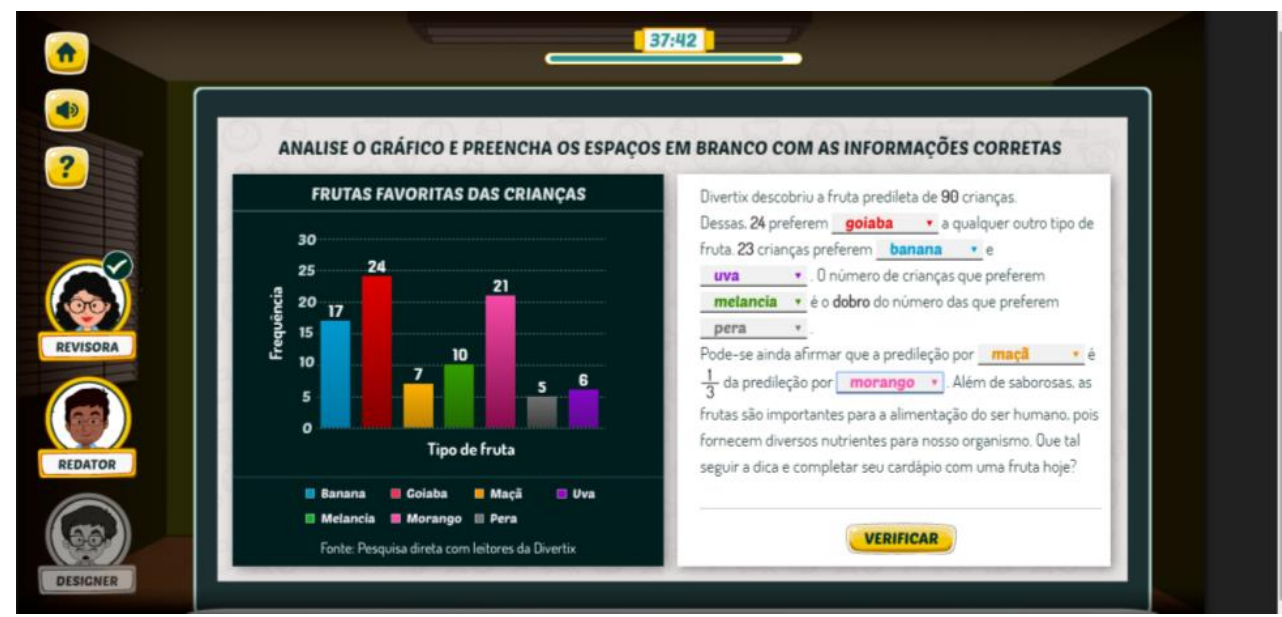

Figura 03. Atividade do Redator

Novamente o jogador irá visualizar um gráfico e um texto, mas, dessa vez, o texto terá campos em branco que deverão ser preenchidos com os nomes das frutas que aparecem no gráfico. Assumindo o papel de redator, o jogador deve verificar, conforme os termos presentes no texto e os dados do gráfico, quais informações preenchem as lacunas corretamente. Caso o usuário verifique que os dados de algumas lacunas não estão compatíveis com o gráfico, ele poderá modificar as lacunas a qualquer momento, até encontrar a resposta correta. Ao finalizar a atividade do revisor, o jogador consegue recuperar mais uma parte da matéria e encontrar uma segunda pista da identidade do vilão.

Subindo mais um andar, o jogador iniciará a atividade do Designer. Nessa atividade o jogador será apresentado a uma matéria sobre esportes (Figura 04). Assumindo o papel de designer, o jogador deverá compor um gráfico de acordo com os dados fornecidos na tabela. Para formar o gráfico o jogador precisará analisar o eixo com o nome dos esportes e o eixo com as quantidades de votos e clicar na quantidade que deverá representar. Ao final, o usuário terá criado um pictograma baseado nos dados do quadro. E por fim, encontrará a última pista da identidade do invasor. Em todas as telas de animação do jogo está presente o botão pular para que o jogador que já conhece a narrativa possa seguir rapidamente para as fases. O botão com símbolo de interrogação contém orientações para ajudar na realização da atividade. O Divertix é um RED que ajudará o professor a desenvolver em seus alunos diversas habilidades. Isso tudo combinado a uma aula mais dinâmica, em que os estudantes poderão aprender se divertindo.

\section{Considerações Finais}

O desenvolvimento do RED, teve como base o modelo iterativo e incremental [Sommerville 2011] porque buscou-se alimentar, em cada uma das etapas, aspectos de cunho pedagógico e técnico, identificados relevantes pela equipe responsável pelo desenvolvimento do recurso. 


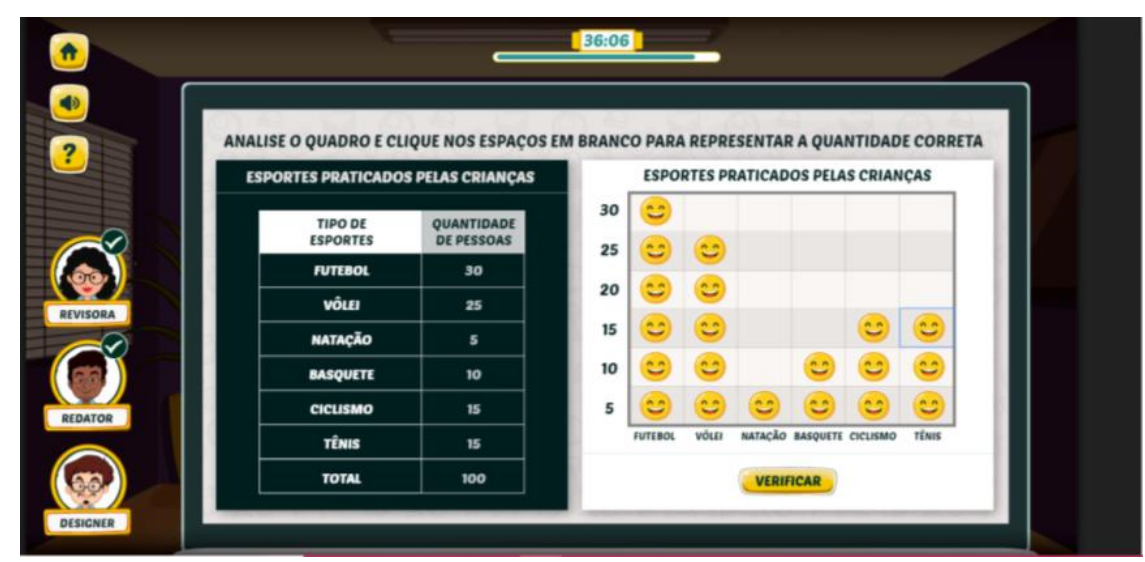

Figura 04. Atividade do Designer

$\mathrm{Na}$ esfera pedagógica, ressalta-se características interdisciplinares, pois permite a ligação da Estatística, unidade temática de Matemática segundo a BNCC, com a Língua Portuguesa, mais precisamente na exploração do campo das práticas de estudo e pesquisa [Brasil 2017a]. Já na esfera técnica, a presença de elementos desafiadores e premiações, buscam trazer maior estimulo aos usuários. Embora o RED não tenha sido validado em condições de sala de aula, espera-se que o mesmo possa viabilizar o desenvolvimento de habilidades de resolução de problemas do campo aditivo e multiplicativo, assim como da capacidade de construir gráficos e de interpretar dados estatísticos em textos, tabelas e gráficos, utilizandose para isso elementos de gamificação que instigam o potencial criativo e fecundo para promover a atenção e o interesse das crianças, possibilitando o desenvolvimento de habilidades relacionadas com a Estatística. Em estudos futuros pretendemos inserí-lo em uma sequência de ensino a fim de verificar o potencial de engajamento e aprendizagem.

\section{Referências}

Brasil. Ministério da Educação. Secretaria de Educação Fundamental. (1997) Parâmetros Curriculares Nacionais: Matemática ( $1^{\circ}$ e $2^{\circ}$ ciclos do ensino fundamental). v. 3, MEC.

Brasil (2017a) "Base Nacional Comum Curricular". Ministério da Educação, Secretaria da Educação Básica, Brasília, DF: SEB. Disponível em: <basenacionalcomum.mec.gov.br>. Acesso em: 24 mar. de 2019.

Brasil (2017b) " SAEB 2017” Ministério da Educação. Brasília, DF: INEP.

Castro, J. B. (2012) "O uso de objetos de aprendizagem para a construção e compreensão de gráficos estatísticos”. Dissertação (Mestrado) - Programa de Pós Graduação em Educação Brasileira, Universidade Federal do Ceará, Fortaleza.

Castro, J. B.; Castro-Filho, J. A. (2015) "Desenvolvimento do Pensamento Estatístico com suporte Computacional. Educação Matemática Pesquisa” (online), v. 17, p. 870-896.

Gal, I. (2002) Adult statistical literacy meanings, components, responsabilities. "International Statistical Review", The Hague, v.70, n. 1, p. 1-25.

Inaf. (2018) Inaf Brasil 2018: resultados preliminares. Instituto Paulo Montenegro em parceria com a ONG Ação Educativa. Disponível em: <https://drive.google.com/file/d/1ez-6jrlrRRUm9JJ3MkwxEUffltjCTEI6/view>. Acesso em: 21 abr. De 2019.

Sommerville, Ian. (2011); Engenharia de Software, 9o ed., São Paulo: Pearson Prentice Hall 